\title{
ONE SENSE OF 'INFORMATION': A quick tutorial to Information-theoretic logic
}

\author{
José M. Sagüillo \\ Department of Logic and Moral Philosophy, University of Santiago de Compostela. Spain \\ E-mail: Josemiguel.saguillo@usc.es
}

\begin{abstract}
One of the multiple meanings of the word 'information' is given implicitly in the postulates and conditions of information-theoretic logic (I-T-L). The tradition of looking at logical phenomena from an informational stance goes back as far as the XIX century. Logicians such as Boole, De Morgan, Jevons, and Venn already suggested that deducing is a sort of unpacking the information already contained in given premises. In the XX century this tradition is recovered by Carnap and Bar Hillel, Cohen and Nagel, and more recently by Corcoran. John Corcoran has articulated a specific information-theoretic viewpoint of logic with its own particular characteristics. I intend to explain the basic ideas of I-T-L by motivating their philosophical underpinnings. One desideratum is to complement and to shed light on some of the philosophical shortcomings of the nowadays paradigmatic model-theoretic concept of logical consequence. Another is to provide a brief sample of questions to be newly addressed form the I-T-L, such as insufficiency as well as redundancy of information in a given axiom-set.
\end{abstract}

Keywords: información, lógica, consecuencia info-teorética, información buena y mala, forma, contenido

\section{Varieties Of Concepts Of Information In Logic And Semantics}

$\mathbf{t}$ is advisable to begin with some comments to show the sense in which Corcoran articulates his ideas in comparison with other twentieth-century authors who also had an informational view sustaining either their accounts of logic, as in Carnap and Bar-Hillel 1952 and Cohen and Nagel 1962/93, or in different senses, sustaining their accounts of semantic content, as in Barwise and Perry 1983, Dretske 1981, and Floridi 2005.

Corcoran gives shape to a logic which explains and develops the unqualified statement that deducing is simply unpacking the information more or less hidden in the premises. In other words, deducing involves information processing in the mind of the thinker. Under this viewpoint, the ontic relation of logical consequence underlying epistemic deductive practice takes propositions to be carriers of information. Let us begin with some examples to illustrate this notion. It is assumed that all arithmetic examples in this paper make use of primitive concepts. Thus, for purpose of illustration, "zero", "one", "two", "successor", "even", "square", etc., are all taken to be primitive concepts.

Perhaps the simplest way of dropping information is by eliminating a conjunct from a conjunction. Thus, the information contained in the proposition "Two is oblong" is already contained in the information of the conjunctive proposition "Two is even and two is oblong". Analogously, perhaps the simplest way of adding information is by introducing a conjunct. The conjunctive proposition "One is square but two is not square" adds the information contained in "One is square" to the information contained in the already given proposition "Two is not square". Disjunction introduction is another usual way of dropping information or avoiding commitment to what was already asserted. In effect, "taking something -but not all- back" is what is 
obtained in asserting the more cautious "I shall visit you in March or April" after having said the more contentful "I shall visit you in March". More dramatically, this maneuver is made more evident when going from "I love you" to "I love you or I used to", which -most probably - lacks information. Likewise, the information in a given disjunction is the information shared by its disjuncts. For example, the information in the proposition "Twelve is oblong" is already contained in the disjunctive proposition "Twelve is odd and oblong or twelve is perfect and oblong". Another simple way of dropping information is by means of a conditional assertion introducing a qualification upon what was already asserted. For example, when passing from "I parked in the faculty lot" to "If I came by car, I parked in the faculty lot". It is easily seen that even less information -in fact no information at all- is conveyed by "If I parked in the faculty parking lot, I parked in the faculty parking lot". Thus, Corcoran's informational viewpoint renders the classical desideratum that a tautology follows from any premise-set, since-lacking information-it can never add information to that already contained in the premises. Likewise, a contradiction logically implies any proposition, since it contains all the information pertinent to the universe considered. Other standard procedures of conveying less or conveying more are obtained by using restrictive and attributive relative clauses. Thus, in the universe of natural numbers, "Every number which is oblong is even" clearly follows from "Every number is even". In contrast, "Every number, which is inductive, is zero or positive" does not follow from "Every number is zero or positive", though of course the converse holds: the latter-"Every number is zero or positive"-does follow from the former"Every number, which is inductive, is zero or positive", which is logically equivalent to "Every number is inductive and is zero or positive".

These simple cases show that the intuition behind the information-based relation of logical consequence is easily reflected in our colloquial and professional argumentative practices. In a more systematic way, in his 1998 , p. 115 , Corcoran provides the natural information-theoretic rendering of logical consequence and logical independence: A premise-conclusion argument is valid if and only if the information in the conclusion is contained in the premise-set. Two propositions are logically equivalent if and only they have the same information. Sharing information content is necessary but not sufficient for the identity of propositions. A premise-conclusion argument is invalid if and only if the information in the conclusion is not (all) contained in the premise-set. In other words, the conclusion of a premise-conclusion argument is independent of the premise-set if and only if the information it contains goes beyond the information contained in the premise-set. A tautology contains no information and a contradiction contains all the information pertinent to the given universe. In addition, in his 2007 , p. 405, Corcoran provides the information-theoretic properties of truth-functional connectives for an appropriate class of propositions pertinent to the class of natural numbers. Thus, we have that the negation of a given proposition contains the information not contained in the given proposition. The information in a conjunction is that of each of its conjuncts. The information in a disjunction is that shared by each of its disjuncts. The information in a conditional is the information in the consequent that is not in the antecedent. It is also straightforward that a universal proposition contains the information of each of its instances and that a particular or existential proposition contains the information shared by each of its instances.

\section{I-T-L in perspective}

Corcoran's information-theoretic logic, which is not his creation but rather an articulation of ideas of thinkers previously mentioned, takes propositions pertaining to a fixed universe as containers of information. Corcoran's viewpoint differs from that of Carnap (1947/60, 25-27) for whom two logically equivalent sentences express one and the same proposition. Thus, for Carnap having the same form is not a necessary condition for the identity of propositions. Corcoran takes any two sentences (of a logically perfect language) having different grammatical forms to express different 
propositions. In this sense, "No even number is odd" is not "No odd number is even", since these two propositions have different subjects and different predicates. More to the point, as Corcoran indicates, having the same information content neither entails nor precludes having the same logical form and conversely. Notice that propositions on this account are abstract entities, each having its own singularity and complexity.

Although each proposition has its own logical form and its own information content, the form itself has no content and the information content itself has no fixed form. One and the same form can receive different information contents. And the information content of one proposition can take on different forms - the forms of any propositions containing it. It is precisely the amorphous character of information that is emphasized to indicate that logically equivalent propositions share the same information. Thus, information is malleable and shaped in different logical forms. As Cohen and Nagel 1962/93 point out, from a purely ontic viewpoint, the information-theoretic concept of logical consequence does not rest on truth-values of propositions but on their information content. They clearly indicate that the relation of logical consequence between propositions is both objective and not determined by truth-value. They are also very careful in distinguishing logical consequence from inference, which is a subjective temporal process presupposing intelligent beings. They certainly point out that logical consequence is formal, but also emphasize that this feature in no way exhausts all there is to say about this relation. Their account of logic presupposes an interpreted language since it is propositions expressed by sentences and not just "marks or sounds" that have information and thus logical consequences. They literally say that interpreted sentences convey information, virtually suggesting that propositions expressed by sentences carry information. Moreover, the delicate balance between the role of content and the role of form in their understanding of logical matters reaches one of their finest points when validity is predicated of concrete arguments composed of propositions in virtue of the relation of implication between premises and conclusion.
This account of validity treats each argument individually; it is opposed to the unfortunate derivative sense in which arguments are valid or invalid in virtue of a general rule or form. From Cohen and Nagel's viewpoint, which Corcoran accepts, the latter viewpoint is exactly backward. According to them, it is the validity of the individual arguments having a given form that gives the form its value.

Contrary to Carnap and Bar-Hillel, Corcoran takes logical validity to be an intrinsic property of an argument to the extent that what determines it is the information contained in the propositions involved and nothing outside the given argument is required. In other words, logical validity is an intrinsic property of an argument on this program. The sense of 'intrinsic' here is illustrated in the discourse of semantic arithmetic. An intrinsic arithmetic property is one that belongs (or does not belong) to a number in virtue of the nature of the number itself. To see the contrast here, we should recall that Carnap and Bar-Hillel in their semantic information theory (1952), building on previous work of Carnap (1942/75, 1947/60, and 1950/67), envisioned an explication for the pre-systematic notion of information content by defining the information content of an interpreted sentence to be the class of possible states of the universe which are excluded by the given statement. In other words, the class of possible states of the universe, in which the given statement is false, provides its information content. In their construction, Carnap and Bar Hillel took possible states of the universe as the designata of their state-descriptions. A statedescription with respect to a given language is a set that contains for each elementary pair composed of an atomic sentence and its negation, one and only one of its components. Notice also that on this account, a tautology has minimum information and a contradiction has maximum information, since a tautology is true in every state and hence it excludes none, whereas a contradiction is false in every state and hence it excludes all. The Carnap and Bar-Hillel insight is not to re-interpret the language, but rather to canvass ways the world could have been according to the means of expression of the [interpreted] language under consideration. In other words, 
the interpretation of the language is kept fixed and the truth-value of a given sentence is determined with respect to alternative states of the world. This suggests classifying Carnap and Bar-Hillel's informational account as extrinsic. Notice that truth in a state is clearly an extrinsic property of a sentence in the sense that it not only depends on what the sentence says but also on how the state is. The sense of the word 'extrinsic' used here is also familiar from semantic arithmetic. In the discourse of semantic arithmetic it is said, for example, that the property of being denoted by a certain numeral is an extrinsic property of a given number. This usage is intuitively clear and widely accepted. Thus, logical implication based on information-content in the present sense is induced on truth values, since it is characterized by the inclusion of the class of states in which the conclusion is false in the class of states in which the premises-set is false. Therefore, Carnap and Bar-Hillel do not have an informational conception of consequence in Corcoran's sense. Rather, starting with a variant of the usual modeltheoretic framework, they are showing how the informational terminology can be accounted for. They are not suggesting, as Corcoran is, that the information content is what users of a sentence grasp.

In a still different sense of the word 'information', Barwise and Perry (1983), and subsequently several others, proposed a different account both of propositions and information. In situation semantics, it is situations that carry information in virtue of making certain states of affairs factual. One may be inclined to think of situations as made up of states of affairs, or perhaps of facts. The relation between facts, states of affairs and situations is rather involved but the important point for present purposes is that in this conception, the informational content of a fact is a true proposition. It is in this sense that this account could be called "neo-Russellian" as opposed to the Cohen and Nagel account and the Corcoran account, which for purposes of comparison, could both be qualified as "neoBoolean". Presumably, information in this setting is, somehow, extracted by intelligent beings from facts, and the information a fact carries is relative to a constraint, which establishes a certain regularity in nature.
Again, for purposes of comparison it should be pointed out (1952) —as John Perry indicates-that situation semantics does not have anything to do with logic per se, as long as logic's main concern is taken to be information processing (personal communication).

It should also be pointed out that information in the Corcoran viewpoint is what propositions contain or what logically equivalent propositions share, but in no case is information said to be contained in concepts. In other words, in this program there is no analogue of meaning compositionality, whereby the information a given proposition contains can be decomposed into the information of each of its individual concepts and vice versa. However, the reader should be aware of other senses of 'information' found in Dretske 1981, p. 45, and in Floridi 2007. These authors share a semantic view in which isolated concepts and not only propositions are said to contain information. This view recovers a sort of neoKantian philosophical standpoint in which, for example, the concept "man" contains the semantic information of the concept "rational". Thus, the proposition "Socrates is a man" implies "Socrates is rational". Notice by contrast that both the Tarskian modeltheoretic concept of logical consequence and the Corcoran information-theoretic concept of logical consequence render the previous argument invalid. In other words, there is an interpretation in which, keeping the same logical form of the previous argument, the premise is true and the conclusion is false. For example, in the universe of natural numbers, "Three is odd" is true and "Three is perfect" is false. Likewise, the information contained in the proposition of the conclusion goes beyond the information contained in the premise. If the given premise-set is expanded with the proposition "Every man is rational" the new argument so obtained is valid under these two concepts of logical consequence. Moreover, information-theoretic logic grants a sense of logical consequence based on information containment of the propositions involved in which fewer concepts or more concepts in a given proposition are not necessarily related with less or more information contained. Any universal 
proposition, such as "Every number is a factor of itself" contains the information of the corresponding universal with a restrictive relative clause, for example as in "Every number that is even is a factor of itself". Adding the new concept "even" does not add information to the given universal proposition. Likewise dropping a restrictive relative clause from a universal proposition never drops but usually adds information to the proposition so obtained.

Related to the previous point is the important issue of whether "information" is factive. It should be clear by now that "information" in the Corcoran sense can be "right" or "wrong" in accordance with both Cohen and Nagel and with Carnap and BarHillel. All these logicians reflect on the familiar deductive experience of processing information contained in either true or false propositions taken as premises and mostly identified in applications of the deductive and the hypothetic-deductive methods. Every successful application of the deductive method establishes for the agent that every consequence of premises all known to be true is also known to be true and every successful application of the hypothetic-deductive method establishes for the agent knowledge that there is at least one false premise when a consequence known to be false is obtained. By contrast, notice that the neo-Kantian trend mentioned above provides support for the factive or positive sense of information, at least to the extent to which the information a concept contains can be said to feature each of its analytical attributes.

Furthermore, there is a sense in which Corcoran's information-theoretic consequence can be said to be modal. If a given proposition logically implies another then it could not be otherwise, to the extent that in this logical relation the identity of the implying proposition and the identity of the implied propositions are both involved. In a similar vein some passages in Cohen and Nagel also suggest that they may have entertained a kind of coextensive use of the information concept of logical consequence together with the necessity and the impossibility concepts of logical consequence. Thus, they often state that the specific task of logic is the study of the conditions under which one proposition necessarily follows from another or under which conditions it is impossible for the consequence to be false with the premises being true, thus making the category of "objective possibility" essential to logical discussion. Again, notice by contrast the different truth-value dependent sense in which the Carnap and Bar-Hillel account can be said to be modal. If the class of designata of statedescriptions is envisioned as providing for ontological possibilities, then it is straightforward to obtain a modal reading of logical implication so defined. Necessarily if the premises are all true the conclusion is true; i.e., in every state in which the premises are true, the conclusion is also true. Similarly, it is impossible for the premises all to be true and the conclusion false: i.e., there is no state in which the premises are all true and the conclusion is false.

This brief survey gives some evidence of the wide variety of meanings in which the word 'information' is being used, and it helps to focus on the philosophical underpinnings of the Corcoran account. Hopefully it provides the reader with the main tenets of the information-theoretic concept of logical consequence, thus eliminating ambiguities and potential misunderstandings.

\section{References}

Bar-Hillel, Y. (1964). Language and Information. Selected essays on their theory and application. Reading Massachusetts: Palo Alto, London, Don Mill, Ontario, Addison-Wesley Publishing Company.

Barwise, J and Etchemendy, J. (1990). Information, Infons and Inference. In Cooper et al (1990), pp. 33-78.

Barwise, J. and Perry, J. 1983. Situations and Attitudes. Bradford Books: M.I.T. Press.

Carnap, R. and Bar-Hillel 1952. An Outline of a theory of semantic information. In Bar-Hillel (1964), pp. $221-274$.

Cooper, R., Mukai, K. and Perry, J. (eds.) (1990). Situation Theory and Its Applications. California: CSLI Publications.

Corcoran, J. (1998). Information-theoretic logic. In Martínez, C., Rivas, U. and Villegas-Forero, L. (1998), pp. 113-135.

CC: Creative Commons License, 2009. 
Corcoran, J. (1999). Information-theoretic logic and transformation-theoretic logic. Included in Ram (1999), pp. 25-35.

Devlin, K. (1991). Logic and Information. Cambridge: Cambridge University Press.

Dretske, F. (1981). Knowledge and the Flow of Information. Oxford: Blackwell.

Floridi, L. (2007). Semantic conceptions of information. The Stanford Encyclopedia of Philosophy (Spring 2007 Edition),

Edward N. Zalta (ed.), [Online] <http://plato.stanford.edu/archives/spr2007/entries/information-semantic/>.

Israel, D. and Perry, J. (1991). What is information? Report no CSLI-91-145.

Ram, M. ed. (1999). Fragments of Science: Proceedings of the Mendel Sachs Symposium. Singapore, New Jersey,

London, Hong-Kong: World Scientific.

Sagüillo, J. M. (1997). "Logical consequence revisited". The Bulletin of Symbolic Logic 3, 216-241.

Sagüillo, J. M. (2008). "Methodological practice and complementary concepts of logical consequence: Tarski and Corcoran". History and Philosophy of Logic (forthcoming).

\section{About the Author}

José Miguel Sagüillo Fernández-Vega, is Full Professor of Logic and Philosophy of Science at the University of Santiago de Compostela in Spain. He has been appointed visiting scholar on several occasions at Oxford (UK), Buffalo (NY-USA) and Berkeley (CA-USA). He is the author of several contributions in the field of mathematical logic and its philosophy, deductive methodology, and argumentation theory. His recent books are El movimiento antimetafísico del siglo XX, with Rom Harré, and El pensamiento lógico-matemático, both published in Madrid: Akal; El arte de persuadir. Algunos elementos de argumentación y retórica, published in Coruña: Ludus. He is also author of several articles published in specialized international journals, such as, Paradoxical argumentations, in Analysis and Evaluation, Logical consequence revisited, in Bulletin of Symbolic Logic, Domains of science, universe of discourse, and omega arguments, in History and Philosophy of Logic, Conceptions of Logical implication, in Logica Trianguli, and Methodological practice and complementary concepts of logical consequence: Tarski's model-theoretic consequence and Corcoran's information-theoretic consequence, also in History and Philosophy of Logic. 